\title{
WIE VERLÄSSLICH IST DAS ARTLENBURG-PRIVILEG ÜBERLIEFERT?
}

\author{
von Detlev Ellmers
}

\section{Abstract: Henry the Lion's Artlenburg Charter (1161)}

The Artlenburg Charter of 1161, only transmitted in later copies, is unquestionably the most hotly disputed document of the twelfth century. While one side views the charter as the founding document of the German Hanse, the other side focusses on the clauses inserted into the charter at a later date, which leads them to a completely different understanding of its significance. Among those in the latter group. Thomas Riis put forward the hypothesis that the Liibeck copy of the charter constitutes nothing less than an augmented version of the document with ,improving additions to the text which was produced around 1225 by the Lübeck canon Marold, which Lübeck presented to Emperor Fredrich II in 1226 - together with a number of other charters which Marold had improved " - in order to obtain the document known to scholars as the ,charter of imperial freedom' (1226). The critical examination of Riis's arguments demonstrates, however, that Lübeck never employed its copy of the Artlenburg charter to argue a point with an external authority, be it in 1226 or at any other subsequent time. This raises the question of why. and for whom, the copy was made. The town seal appended to the charter proves indubitably that it was executed on a mandate from the Lübeck town council. It was this body which ordered the Artlenburg charter (together with Henry the Lion's mandate to the advocate charged with governing the German merchants on Gotland) to be copied into Lübecks Codex of Privileges, which was reserved for confidential use by councillors and merchants. The copy of the charter served to inform them of the conditions under which a permanent, peaceful settlement of the conflicts between Germans and Gotlanders had been reached under the tutelage of Henry the Lion, a settlement which opened trade in the Baltic for merchants sailing from Lübeck. The purpose of the copy was to keep Lübeck's merchants precisely informed of each and every right they enjoyed (since these constituted the foundation of their trade), in order to be able to maintain those rights if 
conflicts arose in the future. Any change to the original text would have been suicidal. Therefore, we can be confident that the text which the copy transmits corresponds to the (lost) original in each and every particular.

Das 1161 in Artlenburg ausgestellte Privileg Heinrichs des Löwen gehört zu den umstrittensten Dokumenten des 12. Jahrhunderts, zumal das Original nicht erhalten ist, sondern sein Text nur in drei gleichlautenden Abschriften überliefert ist. Thomas Riis übernimmt in seiner 2014 vorgelegten Untersuchung' ungeprüft die Hypothese skandinavischer Forscher, dass die Gegenseitigkeitsklausel erst nachträglich in die erste, um 1225 angefertigte Lübecker Abschrift interpoliert worden sei. Als eigenes Ergebnis hält er auch das in derselben Abschrift überlieferte Odelrik-Mandat für ein gleichzeitiges Konstrukt, ,das die deutsche merkantile Präsenz auf Gotland schon zur Zeit Heinrichs des Löwen dokumentieren sollte. ... Die beiden Texte wurden in einer angeblichen Originalurkunde vom Domherrn Marold zusammengestellt und mit anderen Urkunden Kaiser Friedrich II. zur Bestätigung vorgelegt. Das Ergebnis war der Reichsfreiheitsbrief von 1226 " (S. 84). Riis begründet diese kühne Hypothese durch den Schriftvergleich der Abschrift von ca. 1225 mit einer von Marold 1225 geschriebenen Urkunde Bischof Bertolds von Lübeck, die Riis beide abbildet, allerdings mit vertauschten Unterschriften. Er gibt zu, was jeder sieht, dass nämlich das Schriftbild ,recht unterschiedlich“ ist. Trotzdem meint er mit seiner Schriftanalyse gezeigt zu haben, dass Marold beide Texte schrieb (S. 83), „der mehrere frühere Privilegien verbessert" habe, um Lübecks Antrag auf Reichsfreiheit beim Kaiser zu unterstützen (S. 82). Tatsächlich wurde das durch einige Zusätze „verbesserte“ Barbarossa-Privileg von 1188 von zwei namentlich genannten Lübecker Bürgern und einem Lübecker Domherrn dem Kaiser vorgelegt, der es im Mai 1226 bestätigte. $^{2}$ Im Reichsfreiheitsbrief vom Juni 1226 übernahm er daraus einige Passagen und bestätigte noch einmal die Lübeck von Barbarossa verliehenen Rechte. ${ }^{3}$

Thomas Riss, Noch einmal das Artlenburg-Privileg - ein Werk Heinrichs des Löwen oder des Domherrn Marold?, in: Hanse und Stadt. Festschrift für Rolf Hammel-Kiesow zum 65. Geburtstag, hg. von Michael HuNIT und Jan LOKERS, Lübeck 2014, S. 79-84. - Das Artlenburg Privileg: Hansisches Urkundenbuch (im Folgenden: HUB) 1. Nr. 15; auch in: Die Urkunden Heinrichs des Löwen. Herzogs von Sachsen und Bayern, bearb. von Karl Jordan (Monumenta Germaniae Historica. Diplomata regum et imperatorum Germaniae. Laienfürsten- und Dynastenurkunden der Kaiserzeit 1) Weimar 1949, unveränderter Nachdruck Stuttgart 1957 (im Folgenden:UHdL), Nr. 48. S. 68-70.

${ }^{2}$ HUB I, Nr. 204.

${ }^{3}$ Text der Urkunde: Olof. AHLeRs (Hg.), Lïbeck 1226. Reichsfreiheit und friihe Stadt, Lübeck 1976, S. 14-17; auch in: Bernd-Ulrich Hergemöller, Qucllen zur Verfassungsge- 
Nun sagt zwar das Sprichwort: „Wer einmal lügt, dem glaubt man nicht." Aber das weckt höchstens einen Verdacht, ist jedoch keine ausreichende Begründung dafür, dass Marold auch anderes gefälscht habe. Dafür müssen inhaltliche Fakten geltend gemacht werden. In Lübecks Reichsfreiheitsbrief kommt ,die deutsche merkantile Präsenz auf Gotland", die durch ein gefälschtes Odelrik-Mandat angeblich dokumentiert werden sollte (S. 84), jedenfalls nicht zur Sprache. Die einzige Stelle, die Lübecks Ostseehandel wenigstens indirekt anspricht, lautet: Omnes insuper negotiatores fideles, venientes ad Civitatem ipsam sive per terram sive per aquam pro negociationibus suis, salve semper ueniant et secure recedant. Für diesen Passus reichten die nicht interpolierten Angaben des Barbarossa-Privilegs völlig aus. ${ }^{+}$Im Gegensatz zu Riis' Behauptung brauchte Lübeck auch kein zusätzliches „Argument für die Sonderstellung der Travestadt im Vergleich mit den anderen Städten der Region" (S. 84), denn dieser Vergleich fand im Reichsfreiheitsbrief überhaupt nicht statt, sodass auch dafür eine Fälschung überflüssig war. Schließlich gibt es im Reichsfreiheitsbrief keine einzige Formulierung aus dem Artlenburg-Privileg oder dem Odelrik-Mandat. Die beides enthaltende Urkunde lag dem Kaiser demnach gar nicht vor. Die Lübecker erwarteten vom Kaiser Hilfe bei ganz anderen Problemen (z. B. in England) und werden sich gehütet haben, ihn mit einer Dienstanweisung an den advocatum et judicem einer Hanse deutscher Kaufleute zu behelligen.

Wenn Riis' Hauptargument, dass die postulierte Fälschung Lübecks Reichsfreiheitsbrief unterstützen sollte, sich als hinfällig erwies, könnten wenigstens seine Beobachtungen zum Odelrik-Mandat den Fälschungsverdacht erhärten, die er allein mit Abweichungen vom Wortgebrauch der anderen Urkunden Heinrichs des Löwen begründet (S. 82). Tatsächlich gibt es noch mehr Abweichungen als er aufzählt, denn das Mandat ist überhaupt keine öffentlich-rechtliche Urkunde, sondern eine interne Dienstanweisung des Herzogs an jemanden, den er selbst mit einer schwierigen Aufgabe betraute. ${ }^{5}$ Das macht all jene von Riis aufgezählten Verdachtsmomente gegenstandslos, die er aus dem Unterschied zu Heinrichs öffentlich-rechtlichen Urkunden ableitet. Schließlich stellt er infrage,

schichte des Mittelalters (Freiherr vom Stein-Gedächtnisausgabe, Bd. XXXIV). Darmstadt 200(), Nr. 39, S. 266-273.

${ }^{4}$ HUB 1. Nr. 33; Die Urkunden Friedrichs I. bearb. von Heinrich APPEL.T unter Mitwirkung von Rainer Maria HerkenRATH. Teil 4: 1181-1190) (Monumenta Germaniae Historica [Diplomata 4], Diplomata regum et imperatorum Germaniae $=$ Die Urkunden der deutschen Könige und Kaiser; 10, 4). Hannover 1990, Nr. 981. S. 263-267 (im Folgenden DDFI.).

${ }^{5}$ HUB I, Nr. 16, UHdL Nr. 49: super Teuthonicos, quos tibi regendos commisi. Hier spricht der anweisende Herzog von sich in der 1. Person Singular, in den öffentlich-rechtlichen Urkunden dagegen in der I. Person Plural. 
„ob Heinrich der Löwe überhaupt einen deutschen Vogt und Richter im Ausland ohne Genehmigung der örtlichen Behörden hätte ernennen können" (S. 82). Er übersieht dabei wieder den Wortlaut der Quelle, nach dem Heinrich den Odelrik gar nicht als deutschen Vogt und Richter im Ausland einsetzte. Er betraute ihn nur mit der Leitung der Deutschen," und zwar nicht erst auf Gotland, sondern in seiner Stadt Lübeck. Denn schon vor ihrer ersten Ausfahrt von dort nach Gotland gestattete er den deutschen Kautleuten den Zusammenschluss zu der damals dafür allgemein üblichen Fahrgemeinschaft, die man hanse nannte. Die dafür jährlich fällige Gebühr, die auch hansa hieß, zahlten sie Heinrich in Lübeck, bis Barbarossa sie ihnen 1188 erließ. Sie hatten Odelrik zu ihrem nuncius gewählt, den Heinrich ausdrücklich als deren advocatus et judex hestätigte und damit genau die Aufgaben umriss, die jeder olderman einer Hanse wahrnahm. Dessen richterliche Befugnisse auch im Ausland umfassten nur marktrechtliche Streitfälle der Hansegenossen. Deshalb sollte auch Odelrik alle Hals- und Handgerichtfälle ausdrücklich in der Zuständigkeit der Gotländer belassen. ${ }^{8}$ Diese Regelung gehörte zum Grundmuster damaliger

${ }^{6}$ HUB I. Nr. 16. UHdL Nr. 49: super Teuthonicos quos tibi regendos commisi.

${ }^{7}$ HUB I. Nr. 33; DDFI. Nr. 981: ut cum mercibus suis libere eam et redeant per totum dacatum Saxonie absque hamsa.

${ }^{8}$ Als Beleg dafür, dass Heinrich der Löwe dem nur für die Deutschen als Richter eingesetzten Odelrich mit auf den Weg gegeben hat, dass er die Fälle der Blutgerichtsbarkeit unter den Deutschen den Gotländern überlassen und sich auf die niedere Gerichtsbarkeit beschränken soll. ist eine längere Anmerkung unvermeidlich. Sie muss den Wortaut des Mandats, meine sehr wörtliche Übersetzung und erklärende Bemerkungen enthalten: Der Auftrag Heinrichs des Löwen an den von ihm eingesetzten Odelrich steht in HUB I, Nr. 16: Odelrice, sub optentu gracie mee precipio hibi, ut leges, quales Guttonibus in omni regno mei tradidi, tales super Teuthonicos, quos tibi regendos commisi, ommi diligentia observes; scilicet qui capitali sentencia rei fuerint, illam recipiant, qui de truncatione manum etiam sustineant, reliquos vero illorum excessus secundum leges superius prenotatas dijudica. Die möglichst wörtliche Übersetzung lautet: „Odelrich, unter dem Schut\% meiner Gnade schreibe ich dir vor, dass du die Rechte, wie ich sie den Gotländern in meiner ganzen Herrschaft übergeben habe, ebenso über die Deutschen. die zu leiten ich dir anvertraut habe, mit aller Sorgfalt beachtest. Dabei ist klar. welche Angeklagten zum Tode verurteilt werden, jene behalten sie sich vor. welche hinsichtlich des Handabschlagens übernehmen sie ebenfalls. Die übrigen aber ihrer Vergehen entscheide du gemiiß den oben bezeichneten Rechten." - Was Odelrich zu tun hat, steht in der 2. Pers. Sg. (observes) oder im Imp. Sg. (dijudica), die Obliegenheiten anderer stehen in der 3. Pers. Pl. (recipiam und sustineant). Damit können von den beiden ebenfalls im Plural genannten Völkern nur dic Guttones gemeint sein: Heinrich schärft dem Odelrich ein. Fälle von Blutgerichtsbarkeit unter den Deutschen nicht selbst zu vollziehen. sondern den Gotländern zu überantworten, damit nicht ein Eingriff in deren obere Gerichtskompetenz den mühsam erreichten Frieden wieder authebt. Die Unterscheidung zwischen oberem oder Blutgericht und Niedergericht war ein Axiom mittelalterlichen Rechts. Auch in den anderen Außenposten nahmen die dorthin fahrenden deutschen Kaufleute in ihren internen Angelegenheiten nur die niedere Gerichtsbarkeit wahr, z. B. UBStL I. I, Nr. 13 für

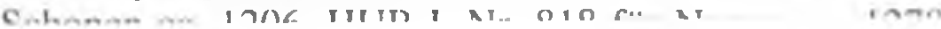


Hansen, sodass es keinen Grund gibt, daraus einen Zweifel an der Echtheit des Mandats abzuleiten.

Bleibt noch die von ihm ungeprüft übernommene Hypothese skandinavischer Forscher, dass die Gegenseitigkeitsklausel erst nachträglich in die erste, um 1225 angefertigte Lübecker Abschrift intcrpoliert worden sei. Schon das Odelrik-Mandat zeigt ja, dass Heinrich den Grundsatz der Gegenseitigkeit auf Gotland durchgeführt wissen wollte. Sieht man sich die Textüberlieferung zum Artlenburg-Privileg genauer an, als es Riis tut, erkennt man auch, für welchen Zweck die Abschriften in Auftrag gegeben wurden. Mit Recht geht Riis für seine Argumentation von der Vorstellung der drei Abschriften aus. Er beginnt mit dem 1368 von Dominikanern und Franziskanern in Visby für Hamburg ausgestellten Transumpt, das nur die Artlenburger Urkunde, aber nicht das Odelrik-Mandat enthält, jedoch ,auch das Privileg vom 11 . Juni 1255 der Grafen Johann und Gerhard von Holstein mitteilt“, an dessen „Echtheit“ Riis nicht zweifelt (S. 79). Obwohl er Wert darauf legt, den Wortgebrauch der jeweiligen Vertragspartner genau zu beachten, tut er das hier nicht, sodass ihm gleich mehrere für seine Argumentation wichtige Zusammenhänge entgehen. Die Grafen nahmen nämlich omnes cives tam in oppido Wishy quam in ipsa terra Gotlandia manentes für den Handel in ihrer Herrschaft in sicheres Geleit und bestätigen ihnen alles Recht, welches sie von ihrem Vater und dessen Vorgängern erhalten haben und wie es durch den Freibrief Herzog Heinrichs von Braunschweig (= Heinrichs des Löwen) beurkundet ist. ${ }^{9}$ Sicheres Geleit und ihr altes Recht ließen sich demnach von Holsteins Grafen diejenigen Bürger bestätigen, deren Siegel die Inschrift: sigillum Theotonicorum in Gotlandia manencium, hatte. Die Gotländer, die sich in ihrem Siegel mit dem Bild des Lammes Gottes als Gutenses signo Christus signantur in agno bezeichneten, waren nicht mit einbezogen. Erst 1280) schlossen sich beide zu der einen Gemeinde Visby zusammen. ${ }^{10}$ Die nach Gotland zugewanderten (manentes) und von dort Handel treibenden deutschen Bürger hatten den Grafen als Beleg ihres alten Rechts folglich das Artlenburg-Privileg Heinrichs des Löwen vorgelegt, dessen Inhalt die Grafen mit dem Verweis auf diesen Freibrief Herzog Heinrichs bestätigten. Das Artlenburg-Privileg war also auch 1255 in Händen der deutschen Bürger Visbys. Weil darauf in der Urkunde von 1255 verwiesen wurde, fügten die Mönche, die davon für Hamburg ein Transumpt anfertigten, auch die Abschrift des Privilegs bei. Der Rat der Stadt Hamburg wollte eine der Urkunden zur Hand haben, die die Sicherheit des Handelsver-

\footnotetext{
${ }^{9}$ HUB I. Nr. 483.

${ }^{10}$ HUB I, Nr. 866.
} 
kehrs auf der durch Holstein führenden Transitstrecke zwischen Hamburg und Lübeck gewährleistete. Das Artlenburg-Privileg kam also unverlangt als Beipack nach Hamburg und spielte dort weiter keine Rolle.

Als zweites Textzeugnis behandelt Riis die um 1225 geschriebene Urkunde, die außer dem Artlenburg-Privileg auch das Odelrik-Mandat enthält. Er nennt sie „Lübecker Redaktion“ (S. 81f.), ohne darauf hinzuweisen, dass der Abschreiber sie ebenso wie 1368 in Überschrift und Schlussbemerkung als Transumpt (Hoc est rescriptum privilegii) bezeichnet und angibt, dass privilegium ipsum repositum est in ecclesia beate Marie virginis in Wisby", die 1225 als Kirche der Deutschen belegt ist. ${ }^{12}$ Insgesamt stimmen also alle drei überlieferten Textzeugen darin überein, dass ein Original des Artlenburg-Privilegs um 1225, 1255 und 1368 bei den deutschen Kaufleuten in Visby lag. Aus unerklärlichen Gründen nennt Riis das Transumpt von ca. 1225 mehrfach ,das angebliche Original des Artlenburg-Privilegs und des Odelrik-Mandats“ (S. 81 zu Abb. 1, S. 82 und S. 84), obwohl doch niemand die Abschriften für die verlorenen Originale gehalten hatte.

Das dritte Textzeugnis ist eine Abschrift von Artlenburg-Privileg und Odelrik-Mandat aus der Urkunde von ca. 1225, die Albert von Bardowik gegen Ende des 13. Jahrhunderts für Lübecks Rat in dessen Codex privilegiorum eintrug. Diese Abschrift hat nach Riis (S. 81) „für die Textkonstitution ... keine Bedeutung", zeigt aber tatsächlich, dass Lübecks Rat den Vertrag von Artlenburg als ein Privileg zu Lübecks Gunsten ansah, an dem er ein so großes Interesse hatte, dass er dessen Transumpt von ca. 1225 als ältestes Privileg für Lübecks Handel in jenen Codex eintragen ließ 3 , den Inhalt also für eine vertrauenswürdige Abschrift echter Urkunden hielt. Lübecks Rat war auch der einzige Rat einer Hansestadt, der für sich das Transumpt selbst anfertigen ließ und es sogar mit allen auf demselben Blatt festgehaltenen Zusatzinformationen durch sein eigenes, erstmals 1223 belegtes Siegel beglaubigte.

Entscheidend für die Beurtcilung dieses Transumpts ist deshalb die Frage, welches Interesse Lübecks Rat am Besitz des Textes hatte, und wofür er ihn gegebenenfalls einsetzte. Dass er ihn 1226 nicht für die Erlangung des Reichsfreiheitsbriefs verwendete, wurde oben schon gezeigt. Auch sonst gibt es in Lübecks reichhaltiger Überlieferung kein einziges Zeugnis dafür, dass der Rat mit Hilfe dieses Textes irgendetwas nach außen zu

"HUB 1. Nr. 15 und 16; UHdL Nr. 48 und 49. Obwohl der letzte Satz unter dem OdelrikMandat steht, kann mit privilegium ipsum nur das Artlenburg-Privileg gemeint sein. denn das Mandat ist kein Privileg.

${ }^{12}$ HUB I, Nr. 191. Urkunde des zustïndigen Bischofs von Linköping: Constructa ecclesia 
bewirken versucht hätte. Die Eintragung in den Codex privilegiorum lässt dagegen das große interne Interesse des Rats an der Kenntnis des Textes erkennen. Die Bedeutung, die das Transumpt für den Rat hatte, ist in den Einleitungsworten zum abgeschriebenen Vertrag unmissverständlich formuliert: Hoc est rescriptum privilegii, quod dedit illustrissimus dux Bawarorum et Saxonum Henricus bene memorie super confirmatione pacis perpetue facta inter Theotonicos et Gutenses. ${ }^{13}$ Dieser von Heinrich dem Löwen erreichte immerwährende Frieden zwischen Deutschen und Gotländern war die unverzichtbare Voraussetzung für die Entfaltung des Ostseehandels deutscher Kaufleute, der deshalb seit 1161 über Lübeck, den damals einzigen deutschen Ostseehafen, unbehindert laufen konnte. Er ermöglichte eine lange andauernde friedliche Zusammenarbeit der Deutschen mit den Gotländern, die u. a. dazu führte, dass die Deutschen ihren Handel auch nach Russland ausdehnen und in Nowgorod eine Handelsstation einrichten konnten. ${ }^{14}$ Kein Wunder, dass die Lübecker Heinrich den Löwen für diese bahnbrechende Unterstützung ihres Handels in guter Erinnerung behielten!

Lübecks Rat hatte das allergrößte Interesse an der Erhaltung dieses Friedens und musste deshalb sehr genau die Bedingungen kennen, unter denen er erreicht wurde. Denn bei aufkommenden Konflikten konnte er nur dann sofort sachgerecht gegensteuern, wenn er die Grundlage seiner eigenen Position detailgenau kannte. Deshalb hatte er keine Veranlassung, dieses Insider-Wissen nach außen zu tragen. Vor allem aber wäre es kontraproduktiv gewesen, diesem Insider-Wissen durch Veränderungen am Text der Originalurkunde die Basis zu entziehen. Deshalb können wir sicher sein, dass die Lübecker Abschrift den Text des in Visbys Marienkirche autbewahrten Originals ganz korrekt wiedergibt. Das wird auch dadurch bestätigt, dass die unabhängig von Lübecks Transumpt 1368 in Visby für Hamburg angefertigte Abschrift des Originals mit dem Lübecker Text übereinstimmt. Im Gegensatz dazu begnügte sich aber der Beauftragte des Lübecker Rats um 1225 in Visby nicht mit der Abschrift des Artlenburger Vertrags, sondern bemühte sich auch noch um sachdienliche Zusatzinformationen, indem er das Odelrik-Mandat abschrieb und auch noch die mündlich dazu gegebenen Mitteilungen notierte. Wenn Riis richtig sieht, dass Marold der Schreiber war, hat dieser mit Umsicht und

${ }^{1.3}$ HUB 1. Nr. 15; UHdL Nr. 48.

${ }^{14}$ HUB I. Nr. 17 und 50) (neues Datum. 1191/92, s. Anna Leonidovna CHorošKEviČ. Der Ostsechandel und der deutsch-russisch-gotländische Vertrag 1191/1192, in: Start JENKS: Michael NORTH ( $\mathrm{Hg}$. ): Der hansische Sonderweg? Beiträge zur Sozial- und Wirtschaftsgeschichte der Hanse (Quellen und Darstellungen zur hansischen Geschichte, N. F.. Bd. 39), Köln u. a. 1993, S. 1-12). 
großer interner Sachkenntnis dem Lübecker Rat ein einmaliges, aber für seinen Bedarf genau passendes Dokument erstellt, dessen Verlässlichkeit der Rat für die nachfolgenden Generationen mit seinem Siegel bestätigte. Nur weil dieses Insider-Wissen sorgfältig dokumentiert wurde, ist mit dem Odelrik-Mandat ausnahmsweise einmal auch eine interne Dienstanweisung Heinrichs des Löwen an jemanden überliefert, den er mit einer besonderen Aufgabe betraut hatte. 